average is a poor picture of the actual behaviour and it is more appropriate to describe the data by a resonance model, but, as the density of resonance increases, it is likely to be a better and better description and the simple Regge model with small numbers of exchanged particles is preferred.

If the fluctuations observed at Argonne are indeed due to the presence of large numbers of overlapping resonances then we have a striking confirmation of the concept of duality and also an indication that the prediotions of explicit quark models for the superabundance of resonances at high masses may be correct.

\section{Magnetic bottles for cosmic rays}

\section{from a Correspondent}

THE interaction of the solar magnetic field with the interplanetary medium and the Earth's magnetic field has been the subject of intense study in recent years by rocket and satellite probes. Solar activity in the form of solar flares gives rise to an increase in the flux of low energy cosmic ray panticles in the vicinity of the Earth. Some solar flares are followed by intense 'magnetic storms' on Earth which cause a reduction in the cosmic ray intensity, referred to as Forbush decreases.

In 1966 cosmic ray detectors aboard the Pioneer 6 and 7 spacecraft found intense fluxes of $\sim 10 \mathrm{MeV}$ charged particles associated with disturbances in the interplanetary medium. The particle fluxes exhibited characteristic pulse profiles with a typical time scale of $\sim 6$ hours. At that time Rao et al. (J. geophys. Res. 72, 4325; 1967) pointed out an almost complete correspondence between such events and the onset of Forbush decreases initiated by solar flares. Moreover the particle fluxes were strongly anisotropic with the direction of anisotropy showing sharp temporal variations. In addition following the decreasing phase of the Forbush decrease a "bidirectional' anisotropy was frequently observed, the two directions of maximum flux being aligned parallel and antiparallel to the interplanetary magnetic field vector.

In 1960 Gold (Astrophys. J. Suppl. 4,406 ) proposed a model for the interplanetary magnetic field associated with solar activity based on magnetic field loops emerging from the Sun's surface and extending to large distances from the Sun ( $~ 20$ solar radii). Such a Gold 'bottle' model received strong support from Skylab observations in 1974 (Gosling \& Roelof Solar Phys. 39, 405).
However Rao et al. (op. cit.) argued that the Gold model could not explain the concurrent observation of both bidirectional and unidirectional fluxes of particles associated with Forbush decreases. They found their observations to be more consistent with the Parker 'blast wave' model in which a shock wave magnetic field disturbance moves out from the Sun. Indeed they argued that only the Parker model could adequately explain their observations.

Further experimental observations have now been reported by Palmer et al. (J. geophys. Res. 83, 75; 1978) and these authors come to the opposite conclusion from Rao et al. Bidirectional anisotropies in low energy solar proton and electron events in the period 196773 have been investigated using data obtained from five satellites, Vela $5 \mathrm{~B}$, $6 \mathrm{~A}$ and $6 \mathrm{~B}$ and Explorers 34 and 41 .

The Vela satellites are in circular orbit at 18 Earth radii distance, inclined at $60^{\circ}$ to the plane of the ecliptic with an orbital period of 112 hours. Protons are detected at 10 different threshold energies in the energy range 0.3 to $45 \mathrm{MeV}$; electrons are detected at four different threshold energies in the range $28-214 \mathrm{keV}$ and electrons of the same energies are detected after being scattered by a gold foil, thereby achieving good discrimination against contaminating proton fluxes. The Explorer satellites are in highly eccentric orbits covering the distance range 1 Earth radius to 35 Earth radii at inclinations of $67^{\circ}$ and $87^{\circ}$ with respect to the ecliptic plane. The Explorers detect protons in the energy range $0.7-125 \mathrm{MeV}$ in five separate energy channels.

Sixteen observations of bidirectional distribution were detected in the 6 year period, including the observation of bidirectionality in three solar electron events. Each on average was of 9 hours duration. The average size of the region in which the bidirectional anisotropy existed was therefore $\sim 0.13$ AU based on a typical value of $600 \mathrm{~km} \mathrm{~s}^{-1}$ for the solar wind speed during disturbed conditions. Some of the observations were very short-lived. Two periods of electron bidirectional anisotropy lasted only 3 to 5 hours each. This implies that the bidirectional anisotropy was restricted to a region of small scale in one dimension. This does not fit with the picture of bidirectional anisotropy associated with large scale magnetic fields which lie between a blast wave and the Sun as suggested by Rao et al. (op. cit.). For agreement to occur the large scale fields must be structured on a much smaller scale.

As with the earlier data Palmer et al. find that the bidirectional anisotropy occurred at the minimum of the associated Forbush decrease in a majority of the events. From this it can be argued that the bidirectionality occurred in a region immediately after the plasma field discontinuity which announced the onset of the Forbush decrease. The authors conclude that a Gold bottle model, or a magnetic regime containing large-scale loops behind an interplanetary shock wave, account best for the observations. This configuration gives rise to a bidirectional anisotropy when energetic particles injeoted from the Sun in one foot of the bottle are mirrored from the other foot. The Gold model accounts for the Forbush decrease minimum which correlates strongly with the bidirectional anisotropy in the solar cosmic rays. The short-lived period observed in energetic electrons indicates that the plasma fields near the front of the driver gas must be structured on a small scale in one dimension. The authors hypothesise that the driver gas consists of loops of field lines in 'shells' which are separated by tangential discontinuities. Thus the Gold bottle model for solar magnetic field disturbances seems to be gaining support.

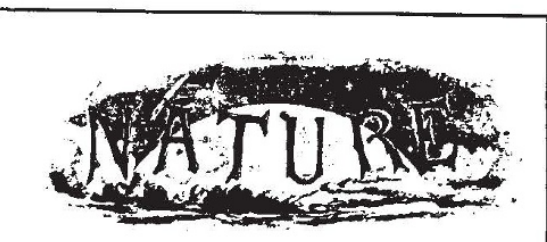

\section{A hundred years ago}

Though the cultivation in India of the best quinine-yielding species of Cinchona $(C$. offricinalis) has not proved a success, it is satisfactory to know that one species at least thrives most abundantly in the Sikkim plantations. From a paper read at the last meeting of the Pharmaceutical Society by Mr Wood, the Government Quinologist in India, it seems that out of a total of about three million trees, comprising four or five species of Cinchona it is estimated that there are as many as 2,500,000 belonging to the species succirubra. It is from this bark that the now wellknown "Cinchona febrifuge" is prepared. This substance, according to many well known medical practitioners in India, possesses to so very nearly the same extent the anti-periodic properties of quinine that it may be safely substituted for the latter in the treatment of ordinary fevers and ague. 5,000 lbs. of this febrifuge, we are told, has already been made and issued, and it is now being made at the rate of 4,000 lbs. a year; the demand, however, is so rapidly overtaking this scale of production that a further extension will shortly be necessary. For use it appears in the form of a white powder, which, however, becomes in a short time of a pale buff tint. It does not agglutinate even in the Indian climate. It is freely soluble in weak acids and is readily taken up by lemon-juice, which constitutes a pleasant vehicle for its administration.

From Nature 17, 21 March, 410; 1878. 